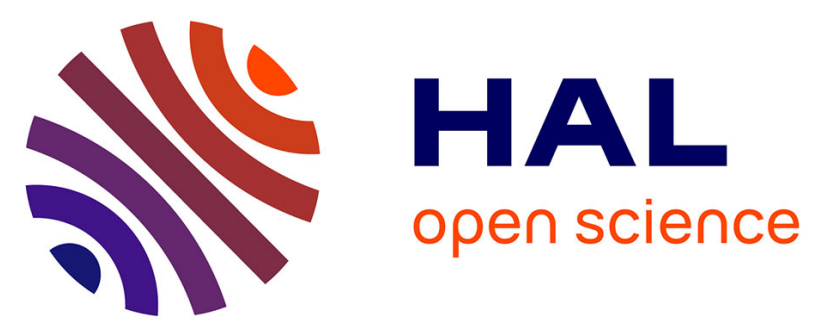

\title{
MESURE DE LA TEMPÉRATURE RÉSIDUELLE DES MATÉRIAUX SOUMIS À UN CHOC INTENSE
}

\author{
M. Mondot, C. Remiot, J. Rigal, P. Breugnot
}

\section{To cite this version:}

M. Mondot, C. Remiot, J. Rigal, P. Breugnot. MESURE DE LA TEMPÉRATURE RÉSIDUELLE DES MATÉRIAUX SOUMIS À UN CHOC INTENSE. Journal de Physique IV Proceedings, 1991, 01

(C3), pp.C3-379-C3-386. 10.1051/jp4:1991354 . jpa-00250498

\section{HAL Id: jpa-00250498 https://hal.science/jpa-00250498}

Submitted on 1 Jan 1991

HAL is a multi-disciplinary open access archive for the deposit and dissemination of scientific research documents, whether they are published or not. The documents may come from teaching and research institutions in France or abroad, or from public or private research centers.
L'archive ouverte pluridisciplinaire HAL, est destinée au dépôt et à la diffusion de documents scientifiques de niveau recherche, publiés ou non, émanant des établissements d'enseignement et de recherche français ou étrangers, des laboratoires publics ou privés. 
MESURE DE LA TEMPÉRATURE RÉSIDUELLE DES MATÉRIAUX SOUMIS ì UN CHOC INTENSE

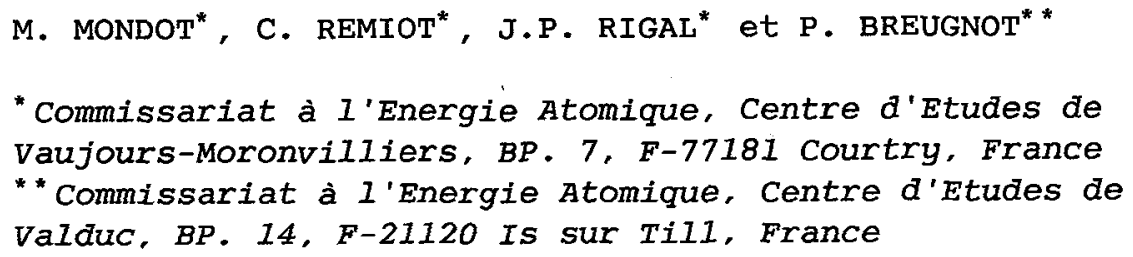

Abstract - We describe an experimental measurement technique which permits to evaluate the free surface temperature of a shock loaded metallic sample. The radiative infrared emission is carried over from the firing chamber to the pyrometer by a fluoride glass fiber. The pyrometer and the measurement technique are described. The free surface temperature of a shock loaded polished aluminum sample is measured and discussed.

\section{INTRODUCTION}

La température des matériaux sous sollicitation dynamique est un paramètre important pour permettre le recalage des équations d'état théoriques. La mesure expérimentale de la température nécessite l'emploi d'un procédé de mesures adapté au phénomène physique concerné. Ainsi, l'emploi de thermocouples placés à l'intérieur du matériau donne accès à une température qui est entachée d'une erreur systématique générée par la modification de la caractéristique statique température-tension du thermocouple lors de la transmission du choc à l'interface matériau-thermocouple. De plus, le temps de réponse des thermocouples, de l'ordre de la microseconde, ne permet pas la détermination précise du suivi temporel de la température. Nous décrivons une chaine de mesure ultrarapide qui permet la détermination de la température vraie d'un matériau soumis à une sollicitation dynamique. Cette chaine de mesure est composée d'un pyromètre infrarouge à fibre optique et d'une centrale d'acquisition et de traitement des données. 


\section{LA TEChNIQUE DE MESURE}

La méconnaissance du facteur d'émission de la plupart des matériaux est le principal problème dans le cadre de la mesure de température par pyrométrie infrarouge /1/.

La théorie du pyromètre bichromatique apporte une solution satisfaisante pour contourner cette difficulté $/ 2 /$. Cependant, la luminance spectrique (formule de Planck) ne caractérise pas la puissance radiative expérimentale parce qu'il n'y a pas d'énergie associée à une longueur d'onde ponctuelle /3/. Pour cette raison, la luminance énergétique (intégration de la formule de planck) est une meilleure solution.

Ainsi, le système de résolution mathématique peut se résumer aux deux équations ci-dessous:

$$
\begin{aligned}
& \mathrm{F}(\mathrm{V} 1)=\epsilon_{1} \cdot \int_{\Delta \lambda_{1}} \mathrm{~L}(\lambda, \mathrm{T}) \cdot \mathrm{d} \lambda \\
& \mathrm{G}(\mathrm{V} 2)=\epsilon_{2} \cdot \int_{\Delta \lambda_{2}} \mathrm{~L}(\lambda, \mathrm{T}) \cdot \mathrm{d} \lambda
\end{aligned}
$$

$\Delta \lambda_{1}$ et $\Delta \lambda_{2}$ sont les bande-passantes optiques de chaque voie de mesure, $V 1$ et $V 2$ sont les tensions mesurées, $F(V)$ et $G(V)$ sont des fonctions de transfert du pyromètre qui permettent d'établir les relations entre le courant électrique débité par le détecteur et le $l$ 'énergie photonique incidente.

A partir de l'hypothèse classique de corps gris, la température vraie $T$ du matériau peut être déduite par itérations successives.

\section{LE PYROMETRE}

Le pyromètre est constitué d'une première partie mobile et amovible qui assure la collection et le transport du flux infrarouge émis par le matériau choqué à l'aide d'une fibre optique /4/ en verre fluoré (figure 1). 


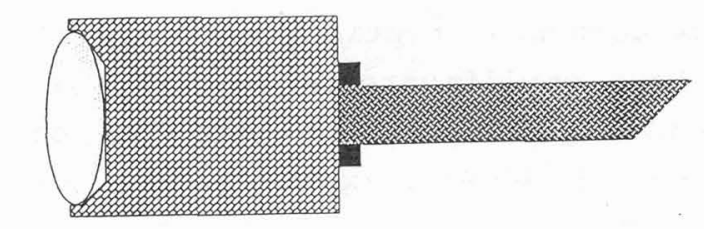

\section{LENTILLE FIBRE OPTIQUE}

Fig 1: Système mobile de collection du flux infrarouge.

La seconde partie du pyromètre assure véritablement la conversion photonique-électrique à l'aide de deux détecteurs photovoltaïques HgCdTe (figure 2).

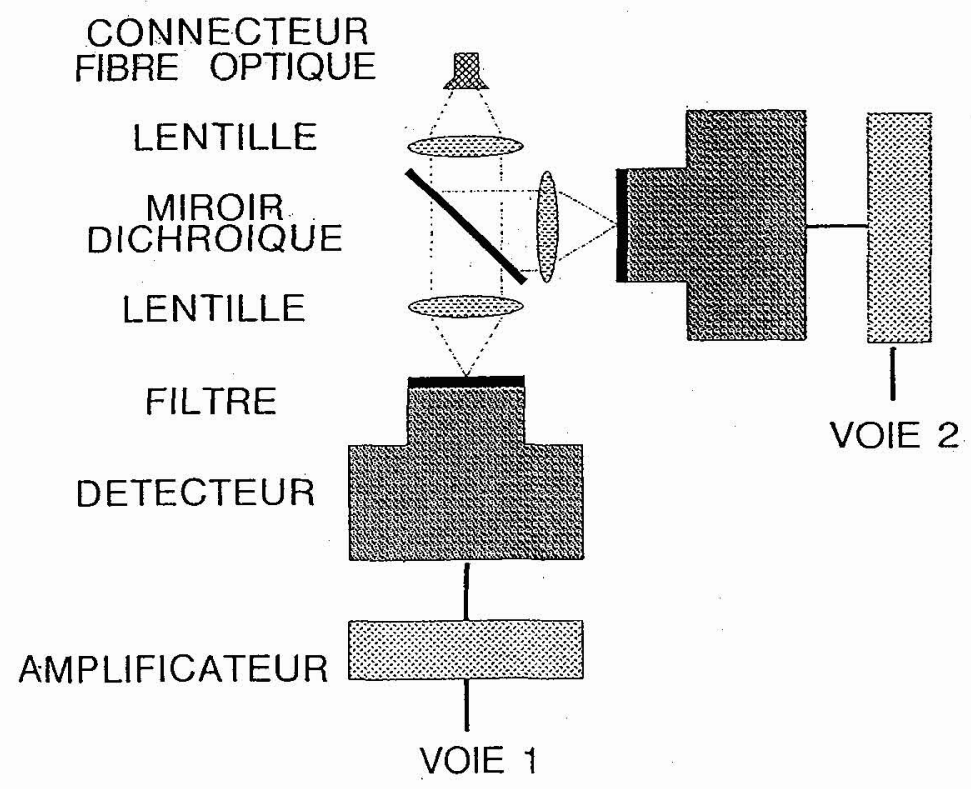

Fig 2: Pyromètre à deux voies de mesure.

Le flux infrarouge est séparé à la sortie de la fibre à l'aide d'un miroir dichroïque puis est focalisé grâce à des lentilles en Znse vers les éléments sensibles des détecteurs. Le domaine spectral utile du pyromètre est compris entre 2 et 5 micromètres. 
Deux filtres optiques étroits, positionnés sur les détecteurs refroidis à la température de l'azote liquide, permettent la sélection de deux domaines spectraux restreints (quelques centaines de nanomètres). Deux amplificateurs assurent le fonctionnement des jonctions photovoltaïques en mode dit de polarisation inverse stabilisé de $1000 \mathrm{~Hz}$ à $500 \mathrm{MHz}$, convertissent le courant débité en tension et amplifient de $45 \mathrm{~dB}$ les signaux obtenus.

Les signaux électriques émis par le pyromètre sont transférés vers la chaine d'acquisition qui est composée d'analyseurs de transitoires pilotés par un micro-ordinateur.

\section{L'ETALOMNAGE DU PYROMETRE}

Le pyromètre est étalonné au moyen d'un corps noir et d'un modulateur mécanique haute fréquence $(5000 \mathrm{~Hz})$. La relation entre la luminance énergétique et la tension est calculée avec un ordinateur pour chaque voie de mesure (figure 3 ):

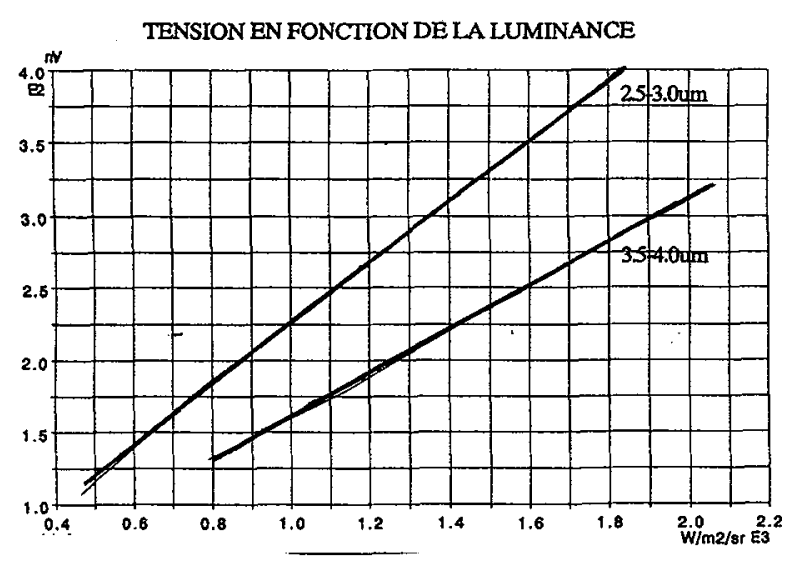

Fig 3: Etalonnage du pyromètre

$$
\begin{aligned}
& \text { Voie 1: } 2.5-3.0 \text { um. } F(V 1)=L\left(\Delta \lambda_{1}, T\right) \\
& \text { Voie 2: } 3.5-4.0 \text { um. } G(V 2)=L\left(\Delta \lambda_{2}, T\right)
\end{aligned}
$$

Il est montré expérimentalement $/ 5 /$ qu'une relation linéaire associe la luminance énergétique de la source à la tension débitée par chaque détecteur. 


\section{IA VALIDATION EXPERIMENTALE}

La validation statique de la chaine de mesure est obtenue par l'étude d'un alliage d'aluminium, AU4G, positionné dans un montage sous vide d'air et chauffé au moyen d'un épiradiateur à une température de $700 \mathrm{~K}$ (figure 4 ):

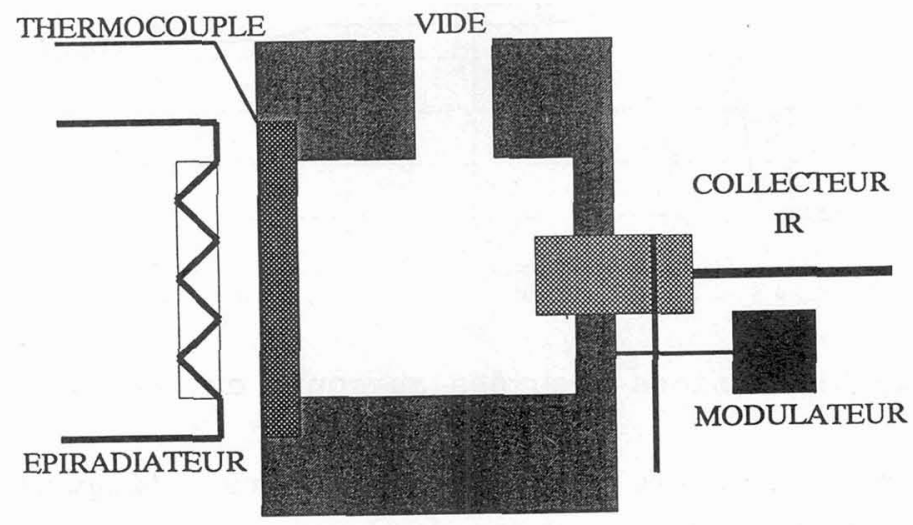

Fig 4: Chauffage statique d'un échantillon sous vide d'air.

La comparaison entre la température mesurée dans le matériau à l'aide d'un thermocouple et celle calculée par la chaine de traitement de données décrite précédemment confirme le bon fonctionnement de la chaine de mesure (pyromètre, acquisition et interprétation).

\section{L'ETUDE DYNAMIQUE DE L'ALUMINIUM}

L'étude dynamique est réalisé au moyen d'un générateur de choc explosif fixé sur un montage identique à celui de l'étude statique précédente; les conditions expérimentales sont:

- une pression de gaz résiduelle d'environ 10-5 mbar,

- une pression de choc de $40 \mathrm{GPa}$,

- un échantillon poli-optique d'alliage d'aluminium AU4G,

- une vitesse de surface libre de $2500 \mathrm{~m} / \mathrm{s}$. 
L'évolution temporelle des signaux est présentée sur la figure 5:

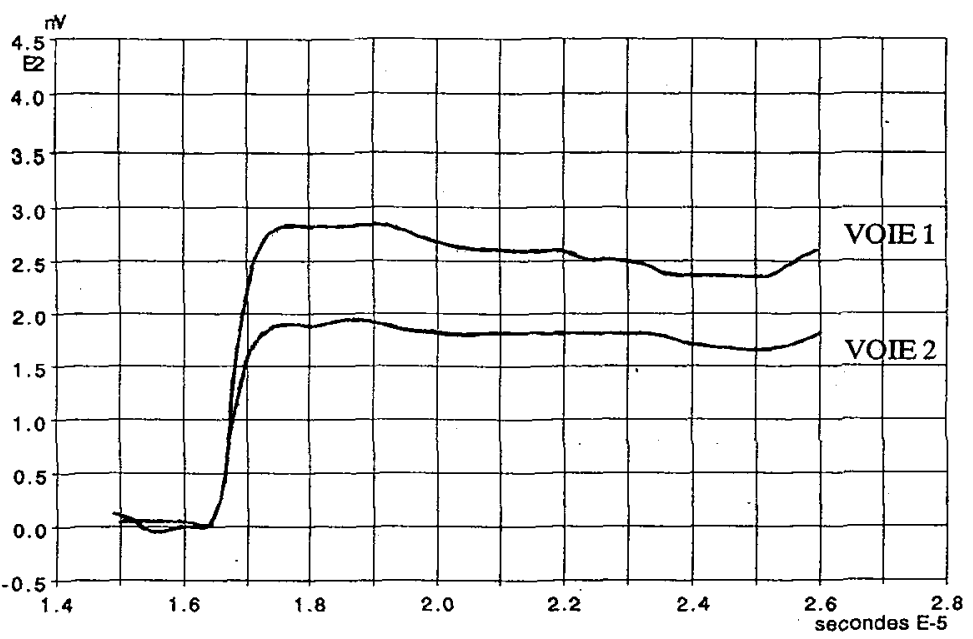

Fig 5: Tensions mesurées au cours du temps.

La dérivée des signaux ci-dessus montre (figure 6) que l'échauffement dure approximativement une microseconde et qu'un palier en température peut être mis en évidence pendant le vol libre de l'échantillon sur quelques millimètres.

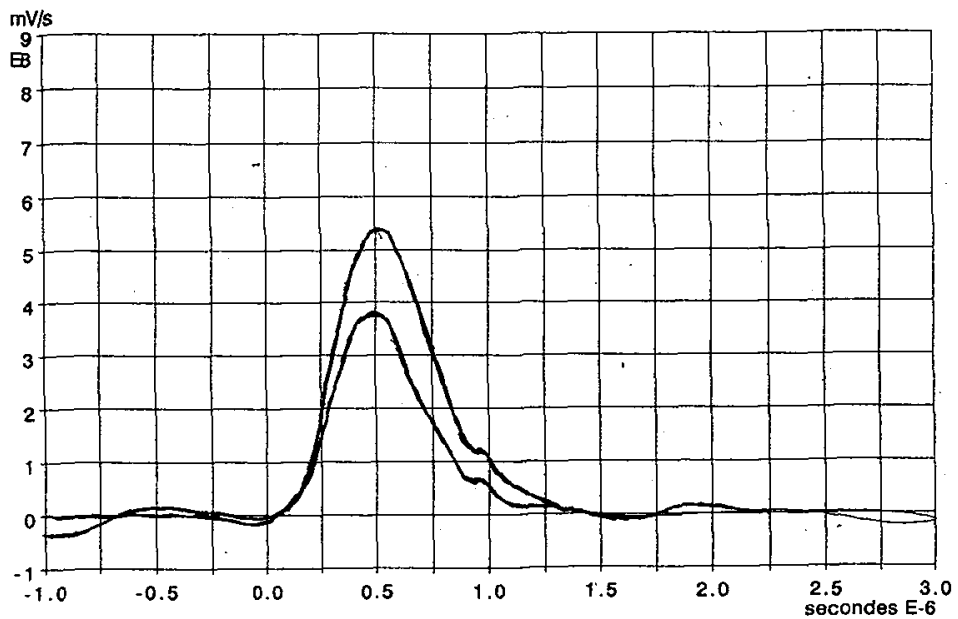

Fig 6: Evolution temporelle de la dérivée des signaux

Le calcul des couples température-émissivité donne les courbes montrées figure 7: 


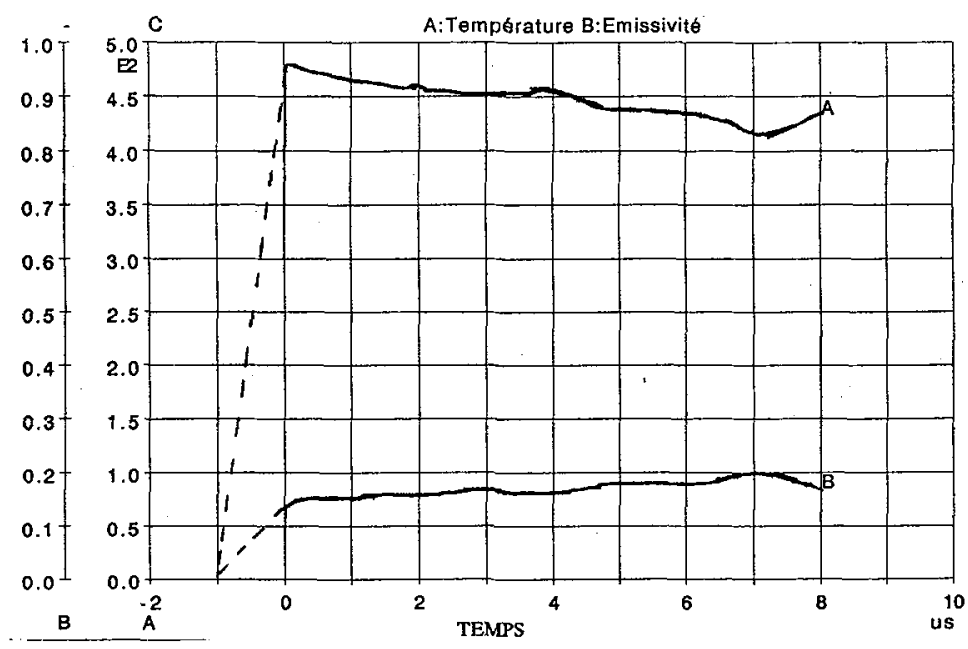

Fig 7: Température et émissivité de l'AU4G choqué à 40GPa.

La température moyenne mesurée, 720K, indique que le matériau n'est pas passé sous forme liquide en détente. Par ailleurs, l'émissivité déduite de 1 'hypothèse de corps gris, 0.15 , confirme ce fait $/ 6 /$. Nous noterons que cette dernière est supérieure à celle déterminée statiquement (environ- 0,04 à $750 \mathrm{~K} / 7 /$ ) parce que la surface libre ne conserve pas un état de surface poli-optique, mais est altérée par l'émergence du choc.

\section{CONCLUSION}

Nous avons montré la faisabilité de la mesure expérimentale des températures résiduelles des matériaux choqués inférieures à $800 \mathrm{~K}$ par une technique jusqu'alors réservée à celles supérieures à 1300K; cette limite étant imposée par la théorie de Planck appliquée au pyromètre bichromatique classique. L'intégration de la loi de planck sur un domaine spectral restreint et la réalisation d'un logiciel permettant la détermination par itérations successives de la température nous fourni un pyromètre qui possède une température de luminance minimale détectable voisine de $500 \mathrm{~K}$, ce qui, compte tenu du temps de réponse de quelques nanosecondes, est très performant. De plus, l'utilisation d'une fibre optique de 15 mètres de longueur permet la mesure dans des montages expérimentaux complexes et difficilement accessibles. 


\section{REF EREICES}

/1/ M. HUETZ AUBERT, J.F. SACADURA

Determination of monochromatic directional reflectivities and emissivities of opaque materials.

Rev. Phys. Appl., V17, N4, p251, 04.82 .

/2/ BOURIANES, MOREAU

Un pyromètre rapide à plusieurs couleurs.

Revue de physique appliquée, Tome 12, p893, 05.77.

/3/ P.J. BECKWITH, K.C.A. CRANE

Two-wavelength infrared pyrometer for large and rapid temperature changes.

Rev. Sci. Instrum., V53, N6, p871, 06.82.

/4/ A. ZUR, A. KATZIR

Use of infrared fibers for low temperature radiometry measurements .

Appl. Phys. Lett. du 17.02.86.

/5/ M. MONDOT

Réalisation d'un pyromètre infrarouge ultra-rapide

à fibre optique

Mémoire Ingénieur CNAM Paris, 05.90.

/6/ VON KLAUS W. LANGE, H. SCHENCK

Messung des spektralen emissionsgrades von mettalen und mettalegierungen.

Archiv fur das Eisenhuttenwesen, 39 Jahrgang, Heft 8 , Gruppe E, N2909, p611, 08.68.

/7/ J.F. SACADURA

Initiation aux transferts thermiques.

LAVOISIER TEC \& DOC 